\title{
A telehealth framework for dementia care: an ADLs patterns recognition model for patients based on NILM
}

\author{
Shuang Dai \\ Department of Mathematical Sciences \\ University of Essex \\ Colchester, UK \\ sd19628@essex.ac.uk
}

\author{
Qian Wang \\ Department of Computer Science \\ Durham University \\ Durham, UK \\ qian.wang173@hotmail.com
}

\author{
Fanlin Meng \\ Department of Mathematical Sciences \\ University of Essex \\ Colchester, UK \\ fanlin.meng@essex.ac.uk
}

\begin{abstract}
The ageing of the population and the increasing number of patients with dementia in modern society undoubtedly put tremendous pressure on the medical system. Providing telehealth care for potential patients and patients with dementia can reduce the burden on both the health system and caregivers. This paper describes a telehealth framework for dementia early detection and dementia care. Specifically, we propose an improved deep neural network model for Non-Intrusive Load Monitoring (NILM), which disaggregates the household's overall energy usage into those of individual appliances based on the sequence-to-point model and transfer learning. The daily behaviour regularities of patients are then inferred by combining principal component analysis and $K$-means clustering based on the disaggregated appliance-level consumptions. Experiments show that the proposed model can significantly improve training efficiency and maintain load disaggregation accuracy, and the inferred behaviour regularities have great potential to be used as useful inputs and prior knowledge to the dementia condition detection platform for early detection and real-time monitoring of patient's conditions.
\end{abstract}

Keywords-non-intrusive load monitoring, dementia, deep neural network, sequence-to-point, transfer learning

\section{INTRODUCTION}

With the aggravation of population ageing, the number of people with dementia ( $\mathrm{PwD}$ ) is growing, increasing pressure on healthcare systems. Dementia progresses from mild to severe. People with mild dementia will suffer from memory loss, and those with severe dementia will need daily functioning support in almost all aspects [1]. Besides, as the pace of human life accelerates, the dependency of PwD further aggravates the pressure of their family carer. Moreover, the elderly who live alone may not even be aware that they have mild cognitive impairment (the early stage of dementia). Therefore, the use of assistive technology for PwD is crucial to keep them safe and healthy [2].

Many assistive technologies have been developed in the last decades [1] [3], varying from reminding devices to activity tracking devices. However, these devices are often tailored to specific application scenarios and rarely consider the preferences of patients. Moreover, many devices need to be intrusively attached to patients or installed in residential envi- ronments, which will have an unpredictable negative impact on patients' emotions since many $\mathrm{PwD}$ tend to reject noticeable devices [4]. Therefore, utilizing assistive technology focusing on self-management and autonomy is more desired to achieve the health care of PwD.

Non-intrusive load monitoring (NILM) using smart meter readings is an ambient intelligence solution for dementia care, which brings benefits from alleviating the assistance burden of caregivers to monitoring the activities of daily life (ADLs) of patients and timely detecting abnormal behaviours of patients, and to alerting the progress of the disease. Smart meter big data are considered as a cost-effective source to achieve NILM with the rolling out of smart meters [5]. Using electricity consumption data (e.g. current, voltage, active power) collected by smart meters to infer ADLs [6] is a practical and commercially feasible method.

This paper introduces a telehealth framework based on NILM to deliver health care supports for PwD, especially at the early stage of the disease (early detection and intervention). We propose an ADLs pattern recognition model as a key part of this telehealth framework. More specifically, our contribution lies in three folds:

- We optimize the structure of a deep neural network based on sequence-to-point and transfer learning to improve the training efficiency of the model while ensuring the disaggregation accuracy.

- Useful data analysis tools such as principal components analysis (PCA) and $K$-means clustering are used based on the disaggregation results of individual household appliances to infer daily routine patterns of PwD.

- The proposed telehealth framework makes it possible to carry out early detection and timely intervention of dementia since it can monitor conditions of both the potential patients and $\mathrm{PwD}$ in real-time.

The remainder of this paper is organized as follows. Section II introduces the related work of existing ambient intelligence solution for dementia care. Section III describes the system framework. The proposed daily activity recognition model is 
introduced in Section IV, followed by the evaluation in Section $\mathrm{V}$. Finally, the conclusion and future work are presented in Section VI.

\section{RELATED WORK}

The existing ambient intelligence solutions for PwD can be categorized into intrusive monitoring and non-intrusive monitoring.

Intrusive monitoring mainly involves wearable sensors such as accelerometers. [7] proposed AlarmNet for assisted-living and residential monitoring, which includes wearable body networks to sense the physiological information of the participants, and intrusive sensors for environmental quality. [8] introduced a ZigBee and ultrasound-based positioning system (ZUPS), which requires patients to wear a physical device consisting of an accelerometer and a button. The ZUPS could locate and detect risky activities of patients. However, intrusive monitoring equipment is cumbersome and expensive to maintain, and its application scope is often limited. In addition, such monitoring devices usually need to be deployed in the home environment, which could cause privacy and ethical issues.

Non-intrusive monitoring, instead, focuses on algorithmic analysis. A popular method of ADLs pattern recognition for PwD is NILM, which utilizes algorithms to derive the state and power consumption of the individual appliance in the patients' homes based on their aggregated smart meter readings. The disaggregated results can then be used for further analysis to detect abnormal behaviours of PwD. Compared to intrusive monitoring which focuses on hardware deployment, non-intrusive monitoring hardware facilities are simple, easy to install and maintain.

Several studies are looking at NILM for healthcare applications. [9] proposed an iterative time-dependent factorial hidden Markov model (FHMM) to perform load disaggregation on appliance-level based on a priori knowledge of general appliance features. They then linked individual appliance usage with ADLs for e-health. [10] investigated the abnormal behaviours through power consumption data, and the remote nursing of the elderly living alone has been studied. The experimental results showed that this method has the advantages of easy deployment, low cost and non-intrusion. [11] monitored ADLs by establishing daily activity indicators to explore the connection between the state of household appliances and human activities. [12] adopted smart meter readings to disaggregate the usage of electrical appliances where the periodic logarithmic Gaussian Cox process was used to automatically learn electrical appliance usage patterns and to detect abnormal appliance usage patterns of the elderly living alone to raise intervention alarms. [13] scored daily routines and detected deviations based on Dempster-Shafter theory (DST). They combined reliability beliefs of multiple appliances to get total reliability for all patterns of appliances. Based on DST, the proposed method simulated the uncertainty of individual appliance usage patterns and had satisfying performance.
Although the prediction accuracy of NILM using deep neural network is satisfying, long training time and high computational cost are disadvantages of the training process. Moreover, it is a practical problem worthy of attention to further apply the load disaggregation results to various fields since most of the literature on NILM only focuses on the disaggregation task itself and miss to explore the applicable scope for the disaggregated results. As mentioned above, with the ageing of the population, the number of potential dementia patients and PwD increases. The PwD in the early stage often do not receive much attention and timely treatment due to the long reassessment cycle, and therefore the progression of the disease cannot be very well controlled in time. To this end, in this paper, we first improve the deep neural network structure to improve the training efficiency, and then utilizing the disaggregation results to conduct the non-intrusive monitoring for activities of daily life (ADLs) of PwD, which could also provide early detection and timely intervention for dementia.

\section{SYSTEM FRAMEWORK}

Dementia is not a disease that meets screening criteria and cannot be cured. Research has shown that interventions in the early stages of dementia can effectively slow the disease progression. In the UK, dementia is only reassessed once every two years after diagnosis [14], and this testing cycle is too long to accurately track the progress of the disease, and patients are likely to miss the optimal time for early intervention and the deterioration of the disease can not be well controlled. Consistent remote monitoring of patients through smart meter readings enables fine-grained tracking of the disease progress of $\mathrm{PwD}$, which is useful for early intervention and intervention to delay the deterioration of the disease.

PwDs interact with household appliances differently at different dementia stages. The main symptoms of mild dementia are memory and judgment loss. Patients at this stage have most ADLs abilities, and their behaviour patterns in using household appliances are similar to that of most normal people. However, patients with mild dementia are likely to have abnormal appliance usage patterns occasionally, such as frequent opening and closing of the refrigerator door, and reusing the washing machine. People with moderate dementia are likely to have obvious symptoms and need urgent help from their carers, such as cooking and washing. Patients with late-stage dementia are almost entirely dependent on the carers and lose the ability to live independently. Patients at this stage may lose the ability to eat or walk independently and have very little interaction with household appliances.

The system framework described in this paper aims at inferring ADLs of PwD through NILM, such that health care providers and families can monitor the condition of the potential patients and PwD remotely in real-time to consistently track any disease deterioration and implement timely intervention measures. Moreover, abnormal life behaviour can be detected to trigger the medical care alert. Fig. 1 shows the proposed telehealth framework. 


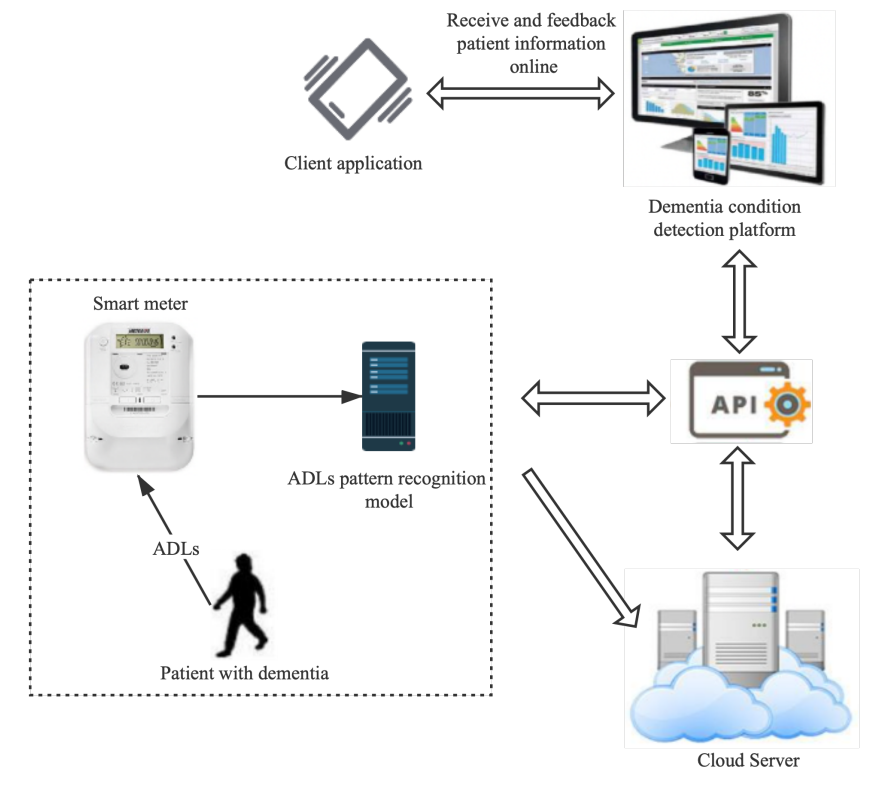

Fig. 1. The proposed general telehealth framework

The aggregated energy consumption of $\mathrm{PwD}$ is summarized by the smart meter and input into the ADLs pattern recognition model. After learning useful signatures for each appliance, the model can disaggregate the target appliances' consumptions related to ADLs of PwD and then summarize the ADLs pattern of patients. Then, the ADLs patterns will be input into the dementia condition detection platform for abnormal behaviours detection. When abnormal behaviours are detected, the platform delivers remote dementia care via an online alert through the client application to the patients and their caregivers. The usefulness of the proposed approach also lies in that the disease progression of $\mathrm{PwD}$ can be monitored through the analysis of changes in the patient's behaviour patterns, which could lead to timely interventions at an early stage to delay the deterioration of dementia.

It is worth pointing out that the acquisition model for ADLs of PwD, namely the ADLs pattern recognition model, plays a crucial role in the system. Considering that accurate load disaggregation remains a recognized challenge, we propose an ADLs pattern recognition model based on an improved NILM architecture in this paper. The disaggregated appliancelevel loads are then further analyzed using PCA and $K$-means to detect and visualize the ADLs of the PwD. The following sections describe the details of ADLs pattern recognition and related methods.

\section{ADLS PATTERN RECOGNITION MODEL}

The ADLs pattern recognition model contains two parts. The first part is an improved NILM architecture based on sequence-to-point and transfer learning. The second part is to apply PCA and $K$-means to detect and visualize the ADLs of the PwD based on the disaggregation results.

\section{A. An improved NILM architecture}

Electrical devices are mainly divided into switch type, finitestate type and continuous variable type [15]. The switching devices only have one status after starting, such as kettles. Finite-state devices allow transitions between multiple states once turned on, such as washing machines and dishwashers. Continuous variable devices have an infinite number of states, such as sewing machines. Due to that the energy consumption of the continuous variable devices is usually insignificant, it is not considered in our framework. The earliest NILM algorithm was based on combinatorial optimization, aiming to determine the best combination state of the monitored electrical appliances and make the total combined power as close as possible to the real meter reading. Deep neural network was firstly applied to NILM by [16] where experiment results indicated its superior performance over traditional methods such as the Factorial Hidden Markov Model (FHMM).

Unlike the traditional deep neural network, the sequenceto-sequence model uses a sliding window to get the neural network input. By dynamically setting a variety of possible sliding window sizes, the output value of the sliding window at each time step is predicted and averaged. This method has been proved to be effective in NILM [16]. However, in the sequence-to-sequence model, the element of the output signal needs to be computed multiple times, which brings redundancy to the calculation. The sequence-to-point model [17] are brought forward to solve this problem. The input of the sequence-to-point model is also a sliding window applied to the main power supply, and the output is a single point from the target appliance. The sliding window ensures that the model learns contextual information of the data, and output is only the midpoint of the window, which can significantly reduce the computational cost during training.

The original model in [18] adopted the convolutional neural network (CNN) to train the model based on the washing machine, which was proved to be able to learn more active channels than other appliances (kettle, fridge, microwave, dishwasher). Then individual consumption patterns of other appliances were fed into the dense layer and combine with the pre-trained CNN to produce the final model to achieve appliance transfer learning. Although this model can achieve satisfactory disaggregation accuracy, it has the same limitations as other deep neural network models such as long training time and high computational cost.

To deal with this, dropout with a rate of 0.5 was used in CNN to improve the generalization ability of the model. Moreover, one-dimensional CNN is used to replace the twodimensional CNN. Besides, we use the trained convolution neural network parameters of the washing machine as the pretrained model for other appliances, and then only trained the dense layer for other appliances to complete transfer learning. Our improved neural network model is expected to accurately conduct NILM for the target appliances from the unseen house and improve the training efficiency of the model. 


\section{B. ADLs pattern recognition}

In order to find out the regularity of daily use of the target appliance of the patients, it is necessary to obtain some important statistics such as the mean power consumption and the usage frequency of each appliance in each hour of a day, and use them as two characteristics to infer the regularity of patients' ADLs.

1) Principle components analysis (PCA): PCA is a mathematical dimensionality reduction method that converts potentially linearly correlated variables into a new set of linearly unrelated variables using orthogonal transformations [19]. PCA is utilized in this paper to make the feature more orthogonal and get the new two-dimensional feature.

2) $K$-means clustering: $K$-means is an iterative clustering algorithm, which divides the data set into $k$ clusters through similarity index evaluation. $K$-means first initializes $k$ centroids representing $k$ clusters, and each data point is assigned to the nearest cluster. The clustering centroid is then updated as the mean vector of all sample points in the updated cluster, and the optimal clustering result will be found through repeated iteration. Due to that $K$-means is simple, and the interpretability of its clustering results satisfies the easy-todistinguish features of the target appliances in this paper, after obtaining the new two-dimensional features via PCA, $K$ means is adopted to cluster the feature set to identify hours in a day when patients are most likely to use each appliance.

\section{Evaluation indicators}

For the comparison and evaluation purpose, mean absolute error (MAE), normalized signal aggregated error (SAE), and $F 1$ score are used to evaluate the performance of the model. Besides, the hit rate (HR) is used to represent the correct classification rate of ADLs pattern recognition.

To test the prediction accuracy at each time point (i.e., the absolute difference between the predicted load $\widehat{y}_{t}$ and the ground truth $y_{t}$ ), MAE is adopted.

$$
M A E=\frac{1}{T} \sum_{t}^{T}\left|y_{t}-\widehat{y}_{t}\right|
$$

In order to evaluate the relative error between the predicted total consumption of the appliance $(\widehat{r})$ and the actual total consumption of the appliance $(r)$, SAE is adopted.

$$
S A E=\frac{|\widehat{r}-r|}{r}
$$

To verify the accuracy of state recognition of each appliance, the $F 1$ score is utilized.

$$
\begin{array}{r}
\text { Precision }=\frac{T P}{T P+F P} \\
\text { Recall }=\frac{T P}{T P+F N} \\
F 1=2 * \frac{\text { precision } * \text { recall }}{\text { precision }+ \text { recall }}
\end{array}
$$

where TP (true positive), denotes the sample is positive and the predicted result is also positive. $F P$ (false positive), predicting negative sample to be positive. $F N$ (false negative), predicting positive samples to be negative. It should be pointed out that the calculation of $F 1$ score needs to use a threshold to determine the ON/OFF status of the target appliance. The threshold values of the switching state of the appliances are obtained directly from the description file in the dataset.

In order to verify the accuracy of ADLs pattern recognition, HR is used.

$$
H R=\frac{h_{c}}{24} * 100 \%
$$

where $h_{c}$ denotes the number of hours of a day that correctly classified ON/OFF states of an appliance.

\section{EVALUATION AND DISCUSSION}

\section{A. Data source and preprocessing}

We chose UK-DALE [20] as the source data set, and the sampling interval of each sub-meter was 6s. To carry out the comparative experiment, we resampled the data to $8 \mathrm{~s}$ to align with the data sampling interval in [18]. The UK-DALE dataset consists of five houses in which the kettle, fridge, microwave, washing machine and dishwasher are commonly used appliances closely related to patients' ADLs (either those with potential dementia or those diagnosed with dementia). Motivated by [16] [17] [21], the target devices selected in this paper include kettle, fridge, microwave, washing machine and dishwasher. Since only houses 1 and 2 in UK-DALE have all these five appliances, we used house 1 as training data to train the improved deep neural network, and implement NILM at appliance-level to the unseen house (i.e., house 2 as test set) via transfer learning.

Precisely, the aggregated reading of the smart meter is taken as the input data of the deep neural network, and the disaggregated load consumption of each appliance is analysed through NILM model, as shown in the following formula

$$
\left[y_{t}^{1}, y_{t}^{2}, \cdots, y_{t}^{n}\right]=f\left(Y_{t}\right)
$$

where $Y_{t}$ denotes the aggregated load at time $t$, the nonintrusive model $f(\cdot)$ disaggregates $Y_{t}$ into individual appliance load consumption. $y_{t}^{i}$ represents the disaggregated load of $i$-th appliance at time $t$, and there are $n$ target appliances used to detect the ADLs pattern.

Abnormal missing data are firstly imputed with mean values. The data are then normalized to facilitate further training of the neural network model.

\section{B. NILM model evaluation}

Different experimental environments have different training costs for the same model. In order to verify the training efficiency of the improved neural network, we crop training data into 100000 samples as input to feed the proposed neural network and the original neural network [18] respectively. Percentages of time and parameters saved by the new model (trained on the washing machine) are shown in Table I.

It can be seen that the improved model significantly saves the training cost of the original model and improves training efficiency. 
TABLE I

NUMBER OF PARAMETERS, TRAINING TIME AND SAVING PERCENTAGES OF THE PROPOSED MODEL COMPARED TO THE ORIGINAL MODEL

\begin{tabular}{|c|c|c|c|}
\hline & \multicolumn{3}{|c|}{ Original model [18] Proposed model Saving percentages } \\
\hline No. of parameters & $10,228,249$ & $1,302,139$ & $87.2 \%$ \\
\hline Training time (s) & 66.13 & 27.67 & $65.7 \%$ \\
\hline
\end{tabular}

The data collected from house 1 in UK-DALE (from November 2012 to November 2013) were used as the training data, and the data from house 2 (from May 20, 2013 to October 10 of the same year) were used as the test data. Fig. 2-Fig. 6 visualize the load disaggregation results of five target devices in the original and the improved models. Table II shows the training results for the improved and original models.

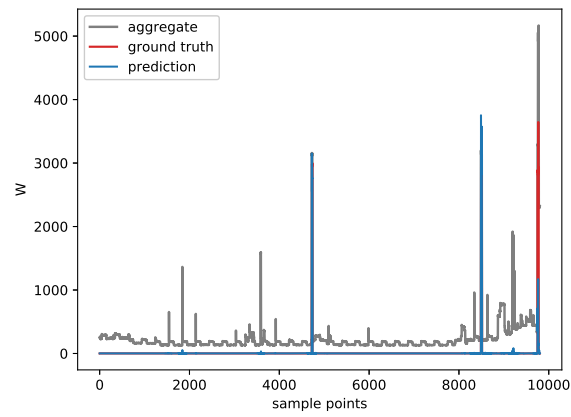

(a) load disaggregation results of the original model

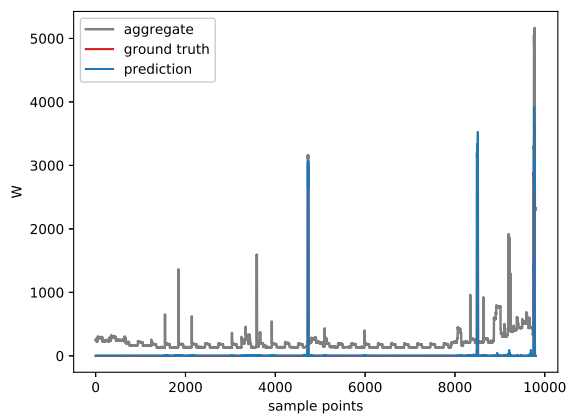

(b) Load disaggregation results of the proposed model

Fig. 2. Load disaggregation results for kettle

TABLE II

TEST RESULTS FOR THE PROPOSED MODEL AND ORIGINAL MODEL; BEST RESULTS ARE MARKED IN BOLD

\begin{tabular}{llllllll}
\hline & \multicolumn{3}{l}{ Proposed model } & \multicolumn{3}{c}{ Original model } \\
\cline { 2 - 7 } & $M A E$ & $S A E$ & $F 1$ & $M A E$ & $S A E$ & $F 1$ \\
\hline Kettle & $\mathbf{9 . 9 0}$ & 0.05 & $\mathbf{0 . 9 1}$ & 10.21 & $\mathbf{0 . 0 3}$ & 0.86 \\
\hline Fridge & $\mathbf{2 0 . 3 6}$ & 0.26 & $\mathbf{0 . 8 1}$ & 25.10 & $\mathbf{0 . 2 1}$ & 0.71 \\
\hline Dishwasher & 23.45 & 0.46 & $\mathbf{0 . 6 9}$ & $\mathbf{1 2 . 8 7}$ & $\mathbf{0 . 0 3}$ & 0.53 \\
\hline Microwave & 14.48 & 0.98 & $\mathbf{0 . 5 3}$ & $\mathbf{7 . 1 4}$ & $\mathbf{0 . 3 5}$ & 0.52 \\
\hline Washing machine & 7.17 & $\mathbf{0 . 3 5}$ & 0.73 & $\mathbf{7 . 1 2}$ & 0.45 & $\mathbf{0 . 8 0}$ \\
\hline
\end{tabular}

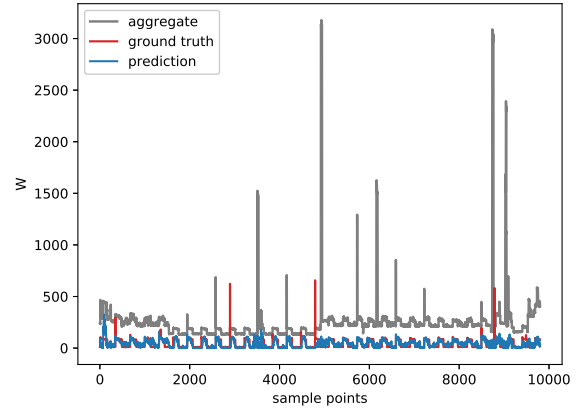

(a) Load disaggregation results of the original model

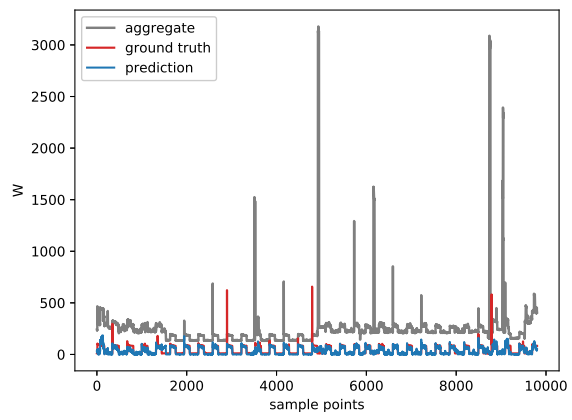

(b) Load disaggregation results of the proposed model

Fig. 3. Load disaggregation results for fridge

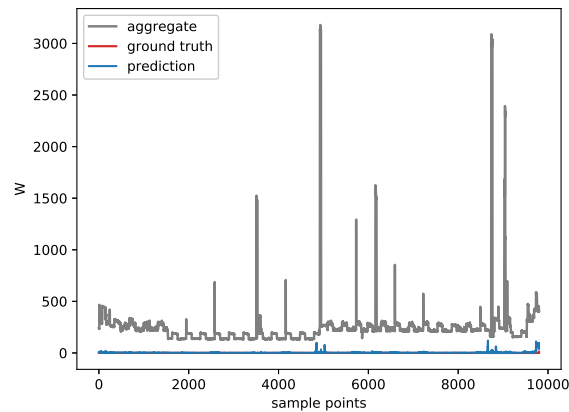

(a) Load disaggregation results of the original model

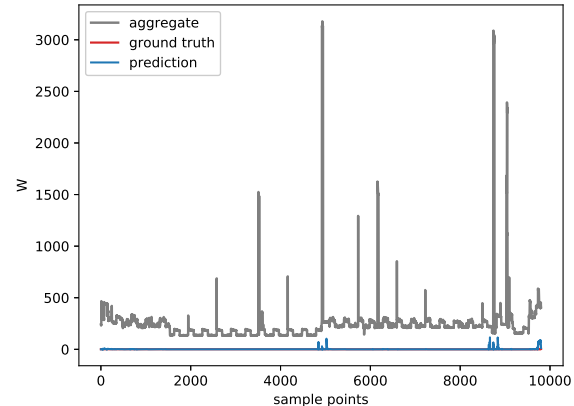

(b) Load disaggregation results of the proposed model

Fig. 4. Load disaggregation results for dishwasher 


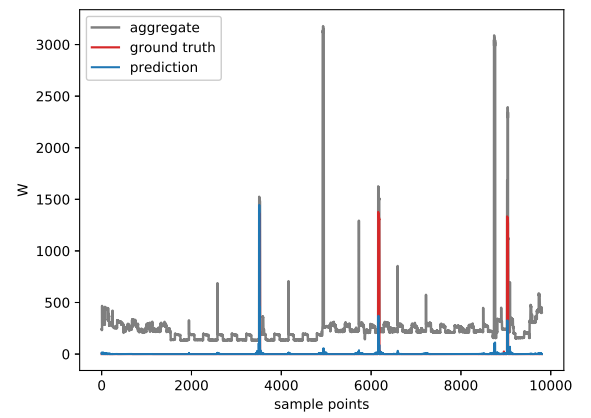

(a) Load disaggregation results of the original model

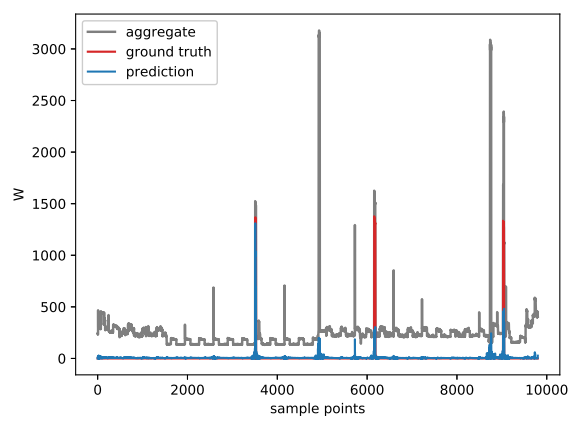

(b) Load disaggregation results of the proposed model

Fig. 5. Load disaggregation results for microwave

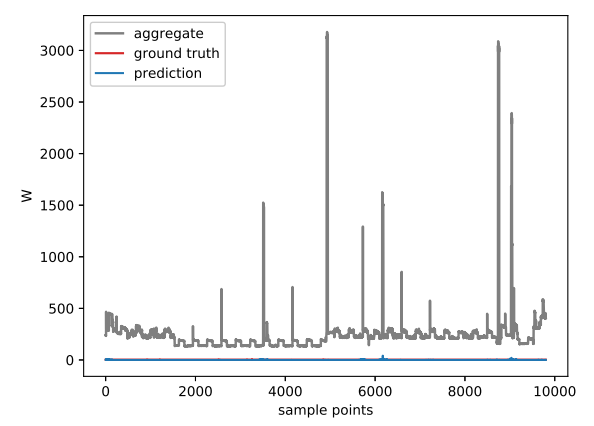

(a) Load disaggregation results of the original model

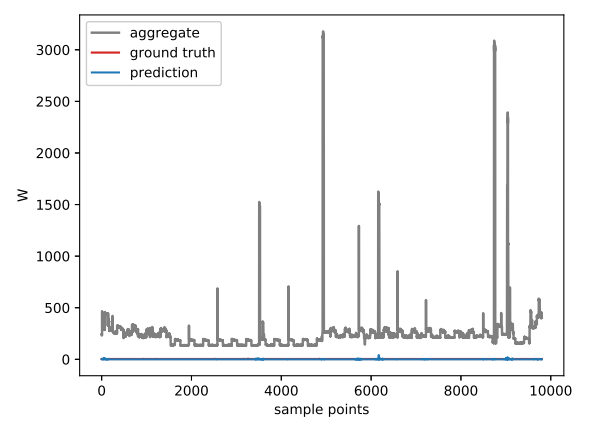

(b) Load disaggregation results of the proposed model

Fig. 6. Load disaggregation results for washing machine
In general, the above comparative results indicate that the improved model could achieve satisfactory disaggregation results with a similar accuracy to the original model. In other words, the improved model is competent for load disaggregation on the premise of greatly saving the training time.

It is worth mentioning that the improved model performs better than the original model in switching devices such as the fridge and kettle. MAE of fridge by the proposed model decreased from 25.10 to 20.36 of the original model, and the visualization results also show that the fitting shape of the proposed model for fridge and kettle is better than that of the original model. On the other hand, more accurate disaggregation results were obtained from the original model for finite-state devices with more complex operation status such as dishwashers, washing machines and microwaves. For the state recognition of each appliance, the improved model is superior to the original model on most of the target appliances (see $F 1$ metrics).

\section{ADLs pattern recognition evaluation}

The dataset we used allows us to calculate the power consumption of the target appliance in the interval of hours. To detect ADLs of PwD, we calculate the mean power consumption and the frequency of power consumption in each hour for each appliance based on the predicted load disaggregation results. Fig. 7 shows the predicted and true use frequency of dishwasher and microwave. The frequency of use is calculated by the ratio of the number of days that the appliance has been used in that hour to the total number of the days considered. The mean power and usage frequency are taken as two features to conduct PCA, and then $K$-means is applied to cluster the new feature set, thus to infer the time of a day when the occupant is most likely to use the target appliance. Table III lists the HR of five target appliances for house 2. To better demonstrate the ADLs pattern recognition performance, Fig. 8 compares the inferred cluster results of dishwasher and microwave with the clustering results based on ground truth data. It can be easily found that for the dishwasher (with a HR of $100 \%$ ), it is mostly likely to be used around the dinner time.

TABLE III

HIT RATE FOR THE TARGET APPLIANCES

\begin{tabular}{ll}
\hline & HR \\
\hline Kettle & $100.00 \%$ \\
\hline Fridge & $75.00 \%$ \\
\hline Dishwasher & $100.00 \%$ \\
\hline Microwave & $66.67 \%$ \\
\hline Washing machine & $95.83 \%$ \\
\hline
\end{tabular}

It can be seen from the Table III that the improved network model can accurately infer the ADLs pattern of participants using kettle, dishwasher and washing machine. However, for fridge and microwave, the inference results still have room to be improved. 


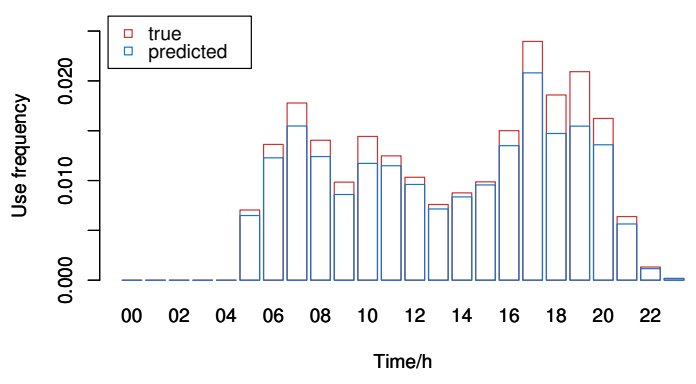

(a) Kettle

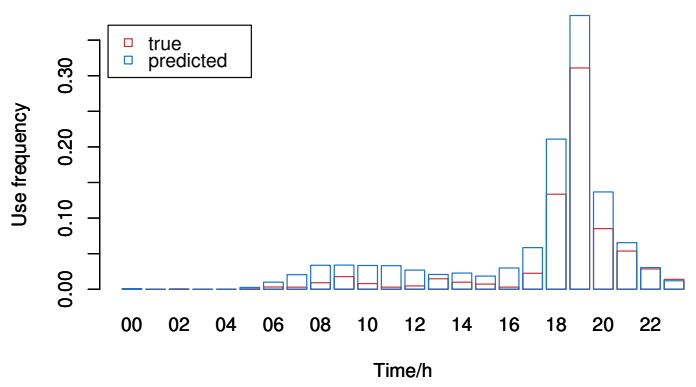

(b) Dishwasher

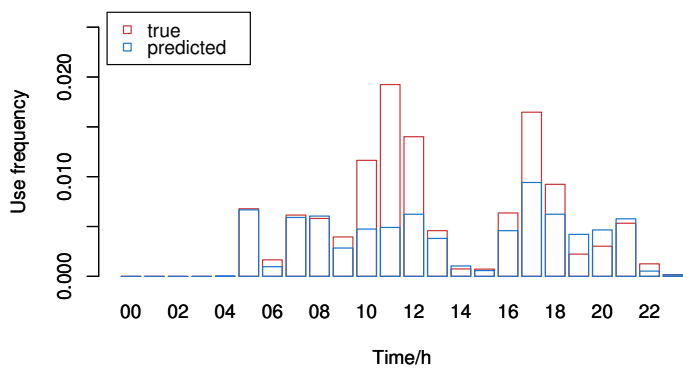

(c) Microwave

Fig. 7. Usage frequency comparison of kettle, dishwasher and microwave

As aforementioned, less accurate disaggregation results for dishwasher and microwave were produced by the proposed model. As can be seen in the Fig. 7, compared with kettle, the predicted use frequency results of both dishwasher and microwave deviated greatly from the ground truth. However, it is interesting to note that the HR for dishwasher reached $100 \%$ whereas the microwave only achieved an HR of $66.67 \%$. One possible reason for this could be that although predicted use frequency for both dishwasher and microwave were mediocre compared with that of kettle, the overall trend of the dishwasher was similar to the ground truth. Instead, the predicted use frequency for microwave was much different from the ground truth. Therefore, the ADLs pattern recognition model may produce less accurate cluster results for microwave usage compared with Dishwasher, as can be seen from Fig. 8.

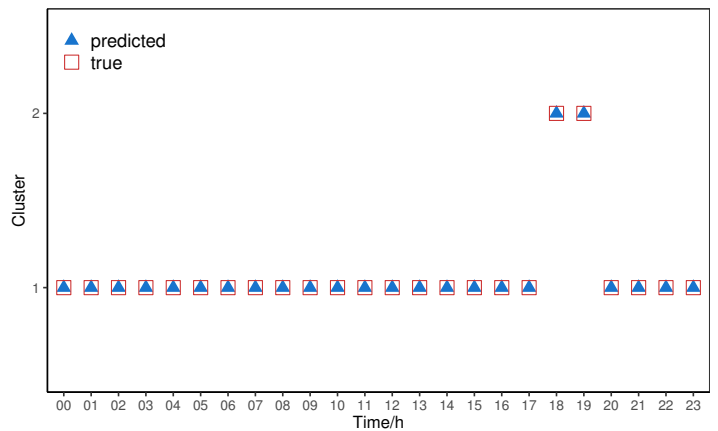

(a) Dishwasher

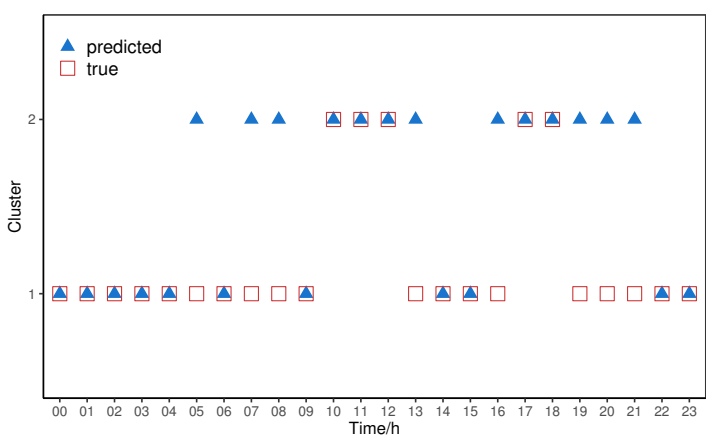

(b) Microwave

Fig. 8. ADLs pattern recognition for dishwasher and microwave: cluster 1 represents the OFF status and cluster 2 represents the ON status

Overall, the proposed model in this paper is useful for performing ADLs pattern recognition based on NILM, which is the focus of this paper. Note that the obtained results based on ADLs pattern recognition can then be used as inputs and prior knowledge to the thereafter dementia condition detection platform. In this way, abnormal behaviour of patients can be monitored, and potential dementia patients can be detected in time, as well as the progress of dementia patients can be consistently followed up, which will be further investigated in our future work.

\section{CONCLUSION AND FUTURE WORK}

This paper introduces a telehealth framework for dementia early detection, real-time monitoring and dementia care based on NILM. More specifically, we propose an improved deep neural network model based on sequence-to-point and transfer learning, which aims to optimize the training efficiency of the model while ensuring the accuracy of the load disaggregation results. Moreover, we utilize useful data analysis tools such as PCA and $K$-means based on the disaggregated appliance consumption to conduct ADLs pattern recognition for potential PwD and PwD. The comparative experimental results show that our improved model can disaggregate usages of target appliances in the unseen house efficiently, and also the usage regularities of target devices can be effectively inferred.

The proposed approach in this paper can be extended in different directions, which will be investigated in our future work. 
1) The collection of data sets. In this paper, the experiments are carried out only on UK-DALE, a public data set. In order to better verify the model proposed in this paper, more real data sets related to dementia patients can be collected in the future, so as to infer the ADLS pattern of patients at various stages of dementia.

2) The selection of switching state threshold. The given switching state threshold is directly collected from the original description file in UK-DALE. However, the actual operating state of the appliance may not always conform to the state threshold given at the time of manufacture. Therefore, clustering and mathematical methods can be developed to select the threshold, such as Variance-Sensitive thresholding (VS) [22] and Activation-Time thresholding [16], so as to identify and monitor the ADLs of PwD more accurately.

3) The anomaly detection technique for the dementia condition detection platform. Based on the ADLs of PwD obtained in this paper, the platform should consistently update the real-time wellbeing of patients under the protocols of privacy protection and alert the abnormal behaviours of patients in time.

\section{ACKNOWLEDGEMENTS}

This work was supported in part by the Challenge Lab Project "Non-intrusive residential energy monitoring for dementia" funded by the ESRC IAA at the University of Essex.

\section{REFERENCES}

[1] S. Lauriks, A. Reinersmann, H. G. Van der Roest, F. Meiland, R. J. Davies, F. Moelaert, M. D. Mulvenna, C. D. Nugent, and R.-M. Dröes, "Review of ict-based services for identified unmet needs in people with dementia," Ageing research reviews, vol. 6, no. 3, pp. 223-246, 2007.

[2] M. Span, M. Hettinga, M. Vernooij-Dassen, J. Eefsting, and C. Smits, "Involving people with dementia in the development of supportive it applications: a systematic review," Ageing research reviews, vol. 12, no. 2, pp. 535-551, 2013.

[3] L. Magnusson, E. Hanson, and M. Borg, "A literature review study of information and communication technology as a support for frail older people living at home and their family carers," Technology and Disability, vol. 16, no. 4, pp. 223-235, 2004.

[4] M. Youssef, M. Mah, and A. Agrawala, "Challenges: device-free passive localization for wireless environments," in Proceedings of the 13th annual ACM international conference on Mobile computing and networking, 2007, pp. 222-229.

[5] A. Adabi, P. Mantey, E. Holmegaard, and M. B. Kjaergaard, "Status and challenges of residential and industrial non-intrusive load monitoring," in 2015 IEEE Conference on Technologies for Sustainability (SusTech). IEEE, 2015, pp. 181-188.

[6] S. Katz, A. B. Ford, R. W. Moskowitz, B. A. Jackson, and M. W. Jaffe, "Studies of illness in the aged: the index of adl: a standardized measure of biological and psychosocial function," Jama, vol. 185, no. 12, pp. 914-919, 1963.

[7] A. D. Wood, J. A. Stankovic, G. Virone, L. Selavo, Z. He, Q. Cao, T. Doan, Y. Wu, L. Fang, and R. Stoleru, "Context-aware wireless sensor networks for assisted living and residential monitoring," IEEE network, vol. 22, no. 4, pp. 26-33, 2008.

[8] A. Marco, R. Casas, J. Falco, H. Gracia, J. I. Artigas, and A. Roy, "Location-based services for elderly and disabled people," Computer communications, vol. 31, no. 6, pp. 1055-1066, 2008.

[9] H. Song, G. Kalogridis, and Z. Fan, "Short paper: Time-dependent power load disaggregation with applications to daily activity monitoring," in 2014 IEEE World Forum on Internet of Things (WF-IoT). IEEE, 2014, pp. 183-184.
[10] C. Nordahl, M. Persson, and H. Grahn, "Detection of residents' abnormal behaviour by analysing energy consumption of individual households," in 2017 IEEE International Conference on Data Mining Workshops (ICDMW). IEEE, 2017, pp. 729-738.

[11] N. Noury, M. Berenguer, H. Teyssier, M.-J. Bouzid, and M. Giordani, "Building an index of activity of inhabitants from their activity on the residential electrical power line," IEEE Transactions on Information Technology in Biomedicine, vol. 15, no. 5, pp. 758-766, 2011.

[12] J. Alcalá, O. Parson, and A. Rogers, "Detecting anomalies in activities of daily living of elderly residents via energy disaggregation and cox processes," in Proceedings of the 2nd ACM international conference on embedded systems for energy-efficient built environments, 2015, pp. 225-234.

[13] J. M. Alcalá, J. Ureña, Á. Hernández, and D. Gualda, "Assessing human activity in elderly people using non-intrusive load monitoring," Sensors, vol. 17 , no. 2, p. 351, 2017.

[14] F. La Frenais, R. Bedder, P. Stone, and E. L. Sampson, "Systematic review of prescribing patterns of analgesic medications for older people living in care homes over time," BMJ Supportive \& Palliative Care, vol. 6, no. 3, p. 397, 2016.

[15] G. W. Hart, "Nonintrusive appliance load monitoring," Proceedings of the IEEE, vol. 80, no. 12, pp. 1870-1891, 1992.

[16] J. Kelly and W. Knottenbelt, "Neural nilm: Deep neural networks applied to energy disaggregation," in Proceedings of the 2nd ACM international conference on embedded systems for energy-efficient built environments, 2015, pp. 55-64.

[17] C. Zhang, M. Zhong, Z. Wang, N. Goddard, and C. Sutton, "Sequenceto-point learning with neural networks for non-intrusive load monitoring," in Proceedings of the AAAI Conference on Artificial Intelligence, vol. 32, no. 1, 2018.

[18] M. D'Incecco, S. Squartini, and M. Zhong, "Transfer learning for nonintrusive load monitoring," IEEE Transactions on Smart Grid, vol. 11, no. 2, pp. 1419-1429, 2019.

[19] S. Raschka, Python machine learning. Packt publishing ltd, 2015.

[20] J. Kelly and W. Knottenbelt, "The uk-dale dataset, domestic appliancelevel electricity demand and whole-house demand from five uk homes," Scientific data, vol. 2, no. 1, pp. 1-14, 2015.

[21] M. Zhong, N. Goddard, and C. Sutton, "Latent bayesian melding for integrating individual and population models," in Proceedings of the 28th International Conference on Neural Information Processing SystemsVolume 2, 2015, pp. 3618-3626.

[22] S. Desai, R. Alhadad, A. Mahmood, N. Chilamkurti, and S. Rho, "Multistate energy classifier to evaluate the performance of the nilm algorithm," Sensors, vol. 19, no. 23, p. 5236, 2019. 